\title{
Non-overlapping Domain Decomposition for Modeling Essentially Unsteady Near-wall Turbulent Flows
}

DOI:

10.1016/j.compfluid.2020.104506

10.1016/j.compfluid.2020.104506

\section{Document Version}

Accepted author manuscript

Link to publication record in Manchester Research Explorer

\section{Citation for published version (APA):}

Chikitkin, A. V., Utyuzhnikov, S., Petrov, M. N., \& Titarev, V. (2020). Non-overlapping Domain Decomposition for Modeling Essentially Unsteady Near-wall Turbulent Flows. Computers \& Fluids, 202, 1-9.

https://doi.org/10.1016/j.compfluid.2020.104506, https://doi.org/10.1016/j.compfluid.2020.104506

\section{Published in:}

Computers \& Fluids

\section{Citing this paper}

Please note that where the full-text provided on Manchester Research Explorer is the Author Accepted Manuscript or Proof version this may differ from the final Published version. If citing, it is advised that you check and use the publisher's definitive version.

\section{General rights}

Copyright and moral rights for the publications made accessible in the Research Explorer are retained by the authors and/or other copyright owners and it is a condition of accessing publications that users recognise and abide by the legal requirements associated with these rights.

\section{Takedown policy}

If you believe that this document breaches copyright please refer to the University of Manchester's Takedown Procedures [http://man.ac.uk/04Y6Bo] or contact uml.scholarlycommunications@manchester.ac.uk providing relevant details, so we can investigate your claim.

\section{OPEN ACCESS}




\title{
Non-overlapping Domain Decomposition for Modeling Essentially Unsteady Near-wall Turbulent Flows
}

\author{
Chikitkin, A. ${ }^{1}$, Utyuzhnikov, S. ${ }^{1,2,3}$, Petrov, M. ${ }^{1}$ and Titarev, V. ${ }^{1,4}$ \\ ${ }^{1}$ Moscow Institute of Physics 83 Technology, Dolgoprudny 141700, Russia \\ 2 School of Mechanical, Aerospace 83 Civil Engineering, \\ the University of Manchester, Manchester, M13 9PL, U.K. \\ e-mail: s.utyuzhnikov@manchester.ac.uk \\ 3 Skolkovo Institute of Science and Technology, Nobel St., 3, 143025, Moscow, Russia \\ 4 Federal Research Center "Computer Science and Control" of RAS, Moscow, Russia
}

\section{Abstract}

In near-wall turbulence modeling it is necessary to resolve a thin boundary layer containing high gradients of the solution. An accurate enough resolution of such a layer can take most of the computational time. The situation becomes even worse for unsteady problems. To avoid time-consuming computations, in the present paper a new approach is developed, which is based on a non-overlapping domain decomposition. The boundary condition of Robin type at the interface boundary between domains is constructed by transferring the original boundary condition from the wall. For the first time, recently developed unsteady interface boundary conditions of Robin type are used for the unsteady Reynolds Averaged Navier-Stokes equations. The interface boundary conditions contain a memory term which takes into account

Email address: s.utyuzhnikov@manchester.ac.uk (Chikitkin, A. ${ }^{1}$, Utyuzhnikov, S. ${ }^{1,2,3}$, Petrov, M. ${ }^{1}$ and Titarev, V. ${ }^{1,4}$ ) 
the nonlocal effect in time to be taken into account for essential unsteady problems. In the case of stationary solutions the new approach automatically reduces to the technique earlier developed for the steady problems. The considered test cases demonstrate that the effect of the memory term can be significant for the accuracy of the near-wall domain decomposition. The criteria for importance of the memory term in the interface boundary condition are formulated and confirmed for turbulent flows.

Keywords: domain decomposition; interface boundary condition; wall functions; turbulence; low-Reynolds-number model; unsteady problems;

URANS; large eddy simulation.

\section{Introduction}

Modeling near-wall turbulent flows is traditionally one of the most complicated problems in computational fluid mechanics. Numerical resolution of a turbulent boundary layer requires significant computational resources because of its complex structure. Nearby the wall there is a very thin laminar sublayer due to the damping effect of the wall and zero velocity at the wall. A sufficiently accurate resolution of the laminar sublayer is important for the entire prediction of a near-wall turbulent flow. However, it is quite limited because it requires up to $90 \%$ of computational time [1] even when the Reynolds Averaged Navier-Stokes models (RANS) are used. The RANS models which take into account the effect of the wall are often called the lowReynolds-number (LRN) models. Their use is still ad-hoc and prohibitively 
expensive for industrial applications where serial computations are required for an optimal design. To resolve the problem, there are two principal approaches outlined further.

In the real-life industrial design the approach based on the high-Reynoldsnumber (HRN) RANS models has been used for many years. Such models are isotropic and do not take into account the effect of the wall. They explore the thinness of the laminar sublayer and extended almost to the wall. The effect of the wall is only counted via near-wall Dirichlet boundary conditions called the wall functions usually set at the center of the first near-wall cell. Such boundary conditions are semi-empirical and usually represent different modifications of the analytical solution for a thin plate [2] based on a logarithmic profile (so called the wall law). As noted by Durbin in [3], this approach should be considered as a domain decomposition where the solution in the inner near-wall region is presented by the wall functions. As can be expected from the physics, this approach is very limited because of its nature of construction. The solution may be very sensitive to the mesh especially to the near-wall cell at the which the wall functions are applied. In particular, the approach may fail if the first cell is situated within the laminar sublayer. To resolve this shortcoming, the scalable [4] and adaptive [5] wall functions were proposed. The principle of domain decomposition can be revealed in the numerical and analytical wall functions [6] which are implemented in the numerical approximation of the governing equations near the wall. To the best of our knowledge, there are no wall functions specifically derived for 
essentially unsteady flows. However, as will be shown below steady interface boundary conditions cannot be used for essentially unsteady problems without significant loss of accuracy.

A different near-wall domain decomposition (NDD) approach based on non-overlapping domain decomposition was proposed in [7], [8], [9], [10]. The interface boundary conditions (IBC) are formulated in a differential form there, in contrast to most wall-functions. As shown in [10], these boundary conditions can be derived in quite a general formulation via the theory of potentials [11]. The NDD approach was first implemented to the HRN turbulence models. Then, it was extended to the LRN models in [12]. The technique can be applied to unstructured grids straightforward since the IBC are mesh independent. The only requirement is that the interface boundary should be set approximately at an equal distance from the wall. This can be achieved via selection of a prism-layer grid near the wall that is a common practice in the boundary-layer simulation. The possible extensions of the approach to taking into account nonlocal effects were demonstrated in [13]. In turn, as shown in [14], if the flow is essentially unsteady, then the IBC should be nonlocal in time. The NDD approach was applied to a number of practical problems in [15], [16], [17]. It was demonstrated that the solution is only weakly sensitive to the distance of the interface boundary from the wall, $y^{*}$. Effectively, $y^{*}$ was shown to control the trade-off between the accuracy and computational time. In comparison to the LRN model, the NDD approach reduced the computational time as much as one order of magnitude 
whilst dropping the accuracy by about $1 \%$.

Essentially unsteady turbulent flows cannot be simulated with the RANS models in which any time dependence is lost. The use of large eddy simulation (LES) becomes more and more affordable with the growth of modern computing power. However, this technique is still very expensive for most real-life industrial applications. For some problems the unsteady RANS equations (URANS) proved to be efficient. The NDD approach is based on the transfer of the boundary conditions from the wall to the interface boundary. In the case of URANS, the IBC should be nonlocal in time. As shown in $[14,18]$ for a model equation, the unsteady IBC (UIBC) should contain a memory term which take into account flow history explicitly. The effect of the memory term was recently studied in detail for the test case of a pulsating laminar channel flow in [19]. In this paper, the exact analytical solution was obtained for both the original problem and the case when NDD is used with steady and unsteady interface boundary conditions. It was shown that the unsteady term in the interface boundary conditions can play a crucial role for highly oscillatory flows. This effect can also be important for URANS/ LES decomposition.

The present paper is organized as follows. In section 2 the derivation of UIBC for model equation is described. In section 3 the governing equations based on URANS are presented. Then, we briefly describe the numerical method used for test problems in section 4 . In section 5 details of implementation of the UIBC for URANS equations in the 3D unstructured-mesh 
solver are explained. In section 6 three test problems are considered: pulsating channel flow, pulsating flow around half cylinder and flow with vortex shedding around cylinder.

\section{Interface boundary conditions for URANS equations}

To formulate the IBC for URANS, consider the following equation:

$$
\phi_{t}=L_{y} \phi+R_{h}
$$

where $\phi$ is the appropriate leading variable of the equation, $L_{y}:=\frac{\partial}{\partial y} \mu \frac{\partial}{\partial y}(\mu$ is the viscosity coefficient). This form is common for any URANS equation except the continuity equation under assumption of a thin boundary layer. As noted above, the UIBC can be obtained by a transfer of the boundary conditions from the wall to an interface boundary via the use of thin boundary layer equations (TBLE).

For the sake of simplicity, we assume that the boundary condition for $\phi$ at the wall is homogeneous. As shown in [19], the boundary condition can be transferred from the wall to the interface boundary $y^{*}$. For that we introduce a number of auxiliary boundary value problems (BVP). 
BVP 1:

$$
\begin{aligned}
& L_{y} V_{0}+R_{h}=0, \\
& V_{0}(0)=0, \\
& V_{0}\left(y^{*}\right)=0,
\end{aligned}
$$

and BVP 2:

$$
\begin{aligned}
& L_{y} V=0, \\
& V(0)=0, \\
& V\left(y^{*}\right)=1 .
\end{aligned}
$$

In addition, we further introduce two auxiliary initial BVPs (IBVPs).

IBVP 1:

$$
\begin{aligned}
& W_{0 \mid t}=L_{y} W_{0}, \\
& W_{0}(0, t)=0, \\
& W_{0}\left(y^{*}, t\right)=0, \\
& W_{0}(y, 0)=w_{0}(y),
\end{aligned}
$$

where $w_{0}(y)=\phi(y, 0)-V_{0}(y)-V(y) \phi\left(y^{*}, 0\right)$, 
and IBVP 2:

$$
\begin{aligned}
& W_{t}=L_{y} W-\frac{d \phi_{y^{*}}}{d t} V-\frac{d V_{0}}{d t}, \\
& W(0, t)=0 \\
& W\left(y^{*}, t\right)=0 \\
& W(0, y)=0
\end{aligned}
$$

Then, the solution in the inner region is provided by

$$
\phi=V_{0}+V \phi_{y^{*}}+W+W_{0},
$$

where $\phi_{y^{*}}=\phi\left(y^{*}, t\right)$.

It is clear that operator $L_{y}$ is negative definite in the case of homogeneous boundary conditions. Then, in the inner region there exists the full system of its eigenfunctions $\Psi_{p}$ :

$$
L_{y} \Psi_{p}=-\lambda_{p} \Psi_{p}, \quad p=1,2, \ldots
$$

which is also orthogonal: $\left(\Psi_{m}, \Psi_{n}\right) \equiv \int_{0}^{y^{*}} \Psi_{m} \Psi_{n} d y=\delta_{m n}$, where $m, n=$ $1, \ldots ; \delta_{m n}$ is the Kronecker symbol equal to 1 if $m=n$ and 0 , otherwise; $\lambda_{p}$, a $p$-th eigenvalue that is real and positive. 
Then, the solution to IBVP (4) is given by

$$
W_{0}=\sum_{1}^{\infty} B_{k}(t) \Psi_{k}
$$

Here, see e.g. [20]:

$$
B_{p}=a_{p} \exp \left(-\lambda_{k} t\right), \quad p=1,2, \ldots
$$

and

$$
a_{p}=\left(W_{0}, \Psi_{p}\right) \equiv \int_{0}^{y^{*}} W_{0} \Psi_{p} d y, \quad p=1,2, \ldots
$$

Next, the solution to IBVP (5) reads as

$$
W=\sum_{1}^{\infty} \widehat{C}_{p} \Psi_{p}
$$

where

$$
\begin{aligned}
& \widehat{C}_{p}=-\left(V, \Psi_{p}\right) \int_{0}^{t} \exp \left(-\lambda_{p}(t-\tau)\right) \frac{d \phi_{y^{*}}}{d \tau}(\tau) d \tau= \\
& -\left(V, \Psi_{p}\right)\left[\phi_{y^{*}}(t)-\phi_{y^{*}}(0) \exp \left(-\lambda_{p} t\right)-\lambda_{p} \exp \left(-\lambda_{p} t\right) \int_{0}^{t} \exp \left(\lambda_{p} \tau\right) \phi_{y^{*}}(\tau) d \tau\right] \approx \\
& -\left(V, \Psi_{p}\right)\left[\phi_{y^{*}}(t)-\lambda_{p} \exp \left(-\lambda_{p} t\right) \int_{0}^{t} \exp \left(\lambda_{p} \tau\right) \phi_{y^{*}}(\tau) d \tau\right]+D_{p},
\end{aligned}
$$

and

$$
D_{p}=-b_{p}(t)+b_{p}(0) \exp \left(-\lambda_{p} t\right)+\lambda_{p} \exp \left(-\lambda_{p} t\right) \int_{0}^{t} \exp \left(\lambda_{p} \tau\right) b_{p}(\tau) d \tau
$$




$$
b_{p}=\left(V_{0}, \Psi_{p}\right), \quad p=1,2, \ldots
$$

Thus,

$$
W=-\phi_{y^{*}} \bar{W}+\sum_{1}^{\infty}\left(\Phi_{p}+D_{p}\right) \Psi_{p}
$$

where

$$
\begin{gathered}
\bar{W}=\sum_{1}^{\infty} \bar{C}_{k} \Psi_{k}, \\
\Phi_{p}=\left(V, \Psi_{p}\right) \lambda_{p} \exp \left(-\lambda_{p} t\right) \int_{0}^{t} \exp \left(\lambda_{p} \tau\right) \phi_{y^{*}}(\tau) d \tau, \quad p=1,2, \ldots
\end{gathered}
$$

and

$$
\bar{C}_{p}=\left(V, \Psi_{p}\right) .
$$

Finally, the entire solution in the inner domain is provided by

$$
\phi=V_{0}+\phi_{y^{*}} V-\phi_{y^{*}} \sum_{1}^{\infty} \bar{C}_{k} \Psi_{k}+\sum_{1}^{\infty}\left(\Phi_{k}+D_{k}+B_{k}\right) \Psi_{k}
$$

or

$$
\phi=V_{0}+\bar{V} \phi_{y^{*}}+\sum_{1}^{\infty}\left(\Phi_{k}+D_{k}+B_{k}\right) \Psi_{k}
$$

where

$$
\bar{V}=V-\sum_{1}^{\infty} \bar{C}_{k} \Psi_{k}
$$

It is clear that the inner solution cannot be obtained until $\phi_{y^{*}}$ is known. However, the UIBC can be found without the solution in the outer region. 
For that we differentiate equation (6) with respect to $y$ at $y=y^{*}$ :

$$
\phi^{\prime}=\bar{V}^{\prime} \phi+V_{0}^{\prime}+\sum_{1}^{\infty}\left(\Phi_{k}+D_{k}+B_{k}\right) \Psi_{k}^{\prime} .
$$

Here and further, the prime denotes differentiation.

In the UIBC (7) the unsteady terms corresponding to eigenfunctions

$\Psi_{k} \quad(k=1,2, \ldots)$ represent the memory terms which are nonlocal in time and explicitly contain some history of the flow.

It is worth noting that without the memory terms, the UIBC (7) fully coincides with the steady IBC [7]:

$$
\phi^{\prime}=V^{\prime} \phi+V_{0}^{\prime} .
$$

Thus, we can first solve the IBVP in the outer region with the IBC set at $y=y^{*}$. Then, we can explicitly obtain the solution in the inner region represented by equation (6).

Next, we consider the effect of unsteady terms in the IBC (7) for a number of test cases.

\section{Governing equations}

For numerical experiments we use URANS equations closed with "nonft2" modification of the Spalart-Almaras (SA) model for the turbulent viscosity [21]. The system of equations can be written in the conservation law 
form:

$$
\boldsymbol{U}_{t}+\frac{\partial \boldsymbol{F}_{1}}{\partial x_{1}}+\frac{\partial \boldsymbol{F}_{2}}{\partial x_{2}}+\frac{\partial \boldsymbol{F}_{3}}{\partial x_{3}}=\boldsymbol{S}(\boldsymbol{U})
$$

where $\boldsymbol{U}$ is the vector of conservative variables; $\boldsymbol{F}_{k}$, flux vectors; $\boldsymbol{F}_{k}=\boldsymbol{F}_{k}^{c}-$ $\boldsymbol{F}_{k}^{v}$, where $\boldsymbol{F}_{k}^{c}, \boldsymbol{F}_{k}^{v}$ are the convective and viscous fluxes:

$$
\boldsymbol{U}=\left[\begin{array}{c}
\rho \\
\rho u_{1} \\
\rho u_{2} \\
\rho u_{3} \\
E \\
\rho \tilde{\nu}
\end{array}\right], \boldsymbol{F}_{k}=\left[\begin{array}{c}
\rho u_{k} \\
\rho u_{1} u_{k}+\delta_{1 k} p \\
\rho u_{2} u_{k}+\delta_{2 k} p \\
\rho u_{3} u_{k}+\delta_{3 k} p \\
(E+p) u_{k} \\
\rho \tilde{\nu} u_{k}
\end{array}\right], \boldsymbol{F}_{k}^{v}=\left[\begin{array}{c}
0 \\
\tau_{1 k} \\
\tau_{2 k} \\
\tau_{3 k} \\
u_{\alpha} \tau_{\alpha k}-q_{k} \\
\sigma^{-1} \rho\left(\nu_{l}+\tilde{\nu}\right) \frac{\partial \tilde{\nu}}{\partial x_{k}}
\end{array}\right]
$$

Here, $\tau_{i j}, q_{k}$ are the components of the viscous stress tensor and heat flux vector correspondingly; $\nu_{l}$, laminar kinetic viscosity; $E$, total energy: $E=\rho\left(e+\sum_{k} u_{k}^{2} / 2\right)$ with $e=R T /(\gamma-1)$ for a perfect gas; $\sigma=2 / 3$.

The components of vector $\boldsymbol{S}$ are equal to zero except for the last component: $\boldsymbol{S}(6)=S_{s a}$. The source term of the SA model is defined by the following expressions:

$$
S_{s a}=c_{b 1} \rho \tilde{S} \tilde{\nu}+c_{w 1} f_{w} \rho\left(\frac{\tilde{\nu}}{d}\right)^{2}+c_{b 2} \frac{\rho}{\sigma} \frac{\partial \tilde{\nu}}{\partial x_{k}} \frac{\partial \tilde{\nu}}{\partial x_{k}}
$$


where

$$
\begin{gathered}
\tilde{S}=S+\frac{\tilde{\nu}}{\kappa^{2} d^{2}} f_{v 2}, \quad S=\sqrt{2 W_{i j} W_{i j}}, W_{i j}=\frac{1}{2}\left(\frac{\partial u_{i}}{\partial x_{j}}-\frac{\partial u_{j}}{\partial x_{i}}\right) \\
f_{v 1}=\frac{\chi^{3}}{\chi^{3}+c_{v 1}^{3}}, \quad \chi=\frac{\tilde{\nu}}{\nu_{m o l}}, \quad f_{v 2}=1-\frac{\chi}{1+\chi f_{v 1}}, \quad f_{w}=g\left[\frac{1+c_{w 3}^{6}}{g^{6}+c_{w 3}^{6}}\right]^{1 / 6} \\
g=r+c_{w 2}\left(r^{6}-r\right), r=\min \left(\frac{\tilde{\nu}}{\tilde{S} \kappa^{2} d^{2}}, 10\right)
\end{gathered}
$$

The dynamic viscosity and thermal conductivity are given by $\mu=\mu_{l}+\mu_{t}$, $\lambda=\lambda_{l}+\lambda_{t}$. In turn, the turbulent viscosity is defined by

$$
\mu_{t}=\rho \tilde{\nu} f_{v 1}
$$

The turbulent thermal conductivity is calculated as $\lambda_{t}=c_{p} \mu_{t} / \operatorname{Pr}_{t}$, where the turbulent Prandtl number is constant: $\operatorname{Pr}_{t}=0.85$.

The turbulent variable $\tilde{\nu}$ is set to zero at the wall. For the free stream boundary condition $\tilde{\nu}=3 \nu_{l}$.

\section{Numerical method}

The near wall domain decomposition has been incorporated into the inhouse "FlowModellium" software package [22]. In this code an implicit finitevolume method on an arbitrary unstructured mesh is implemented. In the present work only unstructured hexahedral mesh is used. The governing

equations of form (9) are integrated over each cell producing semi-discrete 
system:

$$
\frac{d}{d t} \boldsymbol{U}_{i}=\boldsymbol{R}_{i}, \boldsymbol{R}_{i}=-\frac{1}{\left|V_{i}\right|} \sum_{l}\left(\boldsymbol{\Phi}_{l i}^{c}-\boldsymbol{\Phi}_{l i}^{v}\right)+\boldsymbol{S}_{i}
$$

Here, $\boldsymbol{U}_{i}$ is the integral average over the cell $i, \boldsymbol{\Phi}_{l i}^{c}$ is the convective (inviscid) numerical flux through the face $l, \boldsymbol{\Phi}_{l i}^{v}$ is the viscous numerical flux through the face $l$.

The convective fluxes are calculated via solution of Riemann problem on each face of the mesh for two values at both sides of the face. These values are produced by reconstruction procedure. We use a 2nd order local one-dimensional TVD reconstruction with minmod limiter [23] and HLLC Riemann solver [24, 25].

Gradients at face centers needed for viscous flux are calculated using vertex values which are weighted sums of $\boldsymbol{U}_{j}$ values in all neighboring cells. It is to be noted that at the boundary faces values of unknowns are set at the face center, and gradients are calculated using special formulas. This is very suitable for IBC implementation.

For time marching, a second order implicit scheme is used. The nonlinear system is solved iteratively using pseudo-time stepping and our modification [26], [27] of the original LU-SGS method [28, 29]. 


\section{Implementation of IBC}

A more general form of the model linear equation can be considered:

$$
\left(k_{0} \phi\right)_{t}=k_{1}\left(k_{2} \phi_{y}\right)_{y}+k_{3}\left(k_{4} \phi\right)_{y}+k_{5} \phi+k_{6}=L_{y} \phi+R_{h}
$$

in interval $\left[0, y^{*}\right]$ with the following boundary conditions:

$$
\begin{aligned}
& l_{y} \phi(0)=l_{0}, \\
& \phi\left(y^{*}\right)=l_{1} .
\end{aligned}
$$

Here, $k_{j}=k_{j}(y, t), R_{h}(y, t)=k_{6}(y, t)$ and $L_{y} \phi$ denotes all the rest terms.

For such model equation it may be more convenient to derive IBC from discretized form of the differential problem for one time step.

Let us consider a 2 nd order finite difference discretization in $y$ of (14) on a uniform mesh $y_{j}=j h, j=0, \ldots, m, h=y^{*} / m$. For the sake of brevity without loss of generality we consider a particular implicit 1st order method for time stepping, a Dirichlet boundary condition at $y=0$, and $k_{0}=1$. Then discrete equations take the following form: 


$$
\frac{\phi^{n+1}-\phi^{n}}{\Delta t}=D_{y} \boldsymbol{\phi}+l_{0}^{n+1} \underbrace{\left[\begin{array}{c}
c_{L} \\
0 \\
\vdots \\
0
\end{array}\right]}_{\boldsymbol{Z}_{L}}+l_{y^{*}}^{n+1} \underbrace{\left[\begin{array}{c}
0 \\
\vdots \\
0 \\
c_{R}
\end{array}\right]}_{\boldsymbol{Z}_{R}}+\boldsymbol{R}^{n+1}
$$

where superscripts $n, n+1$ denote current and next time layer correspondingly. Vectors $\phi^{n}, \phi^{n+1}$ are of length $m-1$ and contain values of the unknowns at the inner grid points:

$$
\begin{aligned}
& \phi^{n}=\left[\phi\left(y_{1}, t^{n}\right), \ldots, \phi\left(y_{m-1}, t^{n}\right)\right]^{T}, \\
& \boldsymbol{R}^{n+1}=\left[R_{h}\left(y_{1}, t^{n+1}, \ldots, R_{h}\left(y_{m-1}, t^{n+1}\right)\right],\right.
\end{aligned}
$$

$D_{y}$ is a matrix of size $(m-1) \times(m-1)$ containing coefficients from finite difference approximation of (14) in all inner nodes, and $c_{l}$ and $c_{R}$ are these coefficients for values at boundary nodes.

By the virtue of linearity, solution can be expressed as a linear combina- 
tion of two auxiliary solutions:

$$
\begin{aligned}
\boldsymbol{\phi}^{n+1} & =\left(I-\Delta t D_{y}\right)^{-1}\left(\boldsymbol{\phi}^{n}+\boldsymbol{R}^{n+1}+l_{0}^{n+1} \boldsymbol{Z}_{L}+l_{y^{*}} \boldsymbol{Z}_{R}\right)= \\
& =\left(I-\Delta t D_{y}\right)^{-1}\left(\phi^{n}+\boldsymbol{R}^{n+1}+l_{0}^{n+1} \boldsymbol{Z}_{L}+0 \cdot \boldsymbol{Z}_{R}\right)+ \\
& +l_{y^{*}}^{n+1}\left(I-\Delta t D_{y}\right)^{-1}\left(0 \cdot \boldsymbol{Z}_{L}+1 \cdot \boldsymbol{Z}_{R}\right)= \\
& =\boldsymbol{W}_{1}^{n+1}+l_{y^{*}}^{n+1} \boldsymbol{W}_{2}^{n+1}
\end{aligned}
$$

From this form it is clear that $\boldsymbol{W}_{1}$ is the numerical solution by the same scheme of the problem with uniform boundary conditions at the right end:

$$
\begin{aligned}
& \left(W_{1}\right)_{t}=L_{y} W_{1}+R_{h}, \\
& W_{1}\left(y, t^{n}\right)=\phi^{n}(y), \\
& W_{1}\left(0, t^{n+1}\right)=l_{0}^{n+1}, \\
& W_{1}\left(y^{*}, t^{n+1}\right)=0 .
\end{aligned}
$$

and $\boldsymbol{W}_{2}$ is solution of the problem with zero initial condition and uniform boundary conditions at the left end:

$$
\begin{aligned}
& \left(W_{2}\right)_{t}=L_{y} W_{2}, \\
& W_{2}\left(y, t^{n}\right)=0, \\
& W_{1}\left(0, t^{n+1}\right)=0, \\
& W_{1}\left(y^{*}, t^{n+1}\right)=1 .
\end{aligned}
$$

Then, differentiating (18) with respect to $y$, we obtain Robin type IBC 
(a discrete analogue of (7)):

$$
\phi^{\prime}\left(y^{*}, t^{n+1}\right)=\left.\left(\boldsymbol{W}_{1}\right)^{\prime}\right|_{y^{*}}+\left.\phi\left(y^{*}\right)\left(\boldsymbol{W}_{2}\right)^{\prime}\right|_{y^{*}}
$$

here $\left.\left(\boldsymbol{W}_{1}\right)^{\prime}\right|_{y^{*}}$ and $\left.\left(\boldsymbol{W}_{2}\right)^{\prime}\right|_{y^{*}}$ are some finite difference approximations to derivative, for example, $\left.\left(\boldsymbol{W}_{1}\right)^{\prime}\right|_{y^{*}}=\left(W_{1, m}-W_{1, m-1}\right) / h$.

Once the coefficients of the UIBC (20) are found, $\phi^{*}$ at the current iteration can be calculated using current value $\phi_{\text {out }}$ in the center of the first neighboring cell in the outer domain (see Figure 1). The derivative in the Robin type boundary condition is replaced with a finite difference:

$$
\frac{\phi_{\text {out }}-\phi^{*}}{h_{1}}=\left.\left(\boldsymbol{W}_{1}\right)^{\prime}\right|_{y^{*}}+\left.\phi\left(y^{*}\right)\left(\boldsymbol{W}_{2}\right)^{\prime}\right|_{y^{*}} \Rightarrow \phi^{*}=\frac{\phi_{\text {out }}-\left.h_{1}\left(\boldsymbol{W}_{1}\right)^{\prime}\right|_{y^{*}}}{1+\left.h_{1}\left(\boldsymbol{W}_{2}\right)^{\prime}\right|_{y^{*}}}
$$

here $h_{1}$ is the distance between cell center and boundary face center.

Figure 1 shows schematically a near-wall region. The red line corresponds to the intermediate boundary, green line shows virtual $1 \mathrm{D}$ grid for numerical solution of the model equation, marker corresponds to the center of cell adjacent to the intermediate boundary.

Value $\phi^{*}$ from $(21)$ is eventually used for computation of viscous and inviscid fluxes at boundary face center. The inviscid flux is calculated via solution of the Riemann problem with $\phi^{*}$ at one side (for each component of $\boldsymbol{U}$ ) and value reconstructed from outer domain at the other side. The viscous flux is calculated directly using gradients obtained with account for $\phi^{*}$. Besides, near-wall profile $\phi(y)$ is also calculated from (18) or (6). 


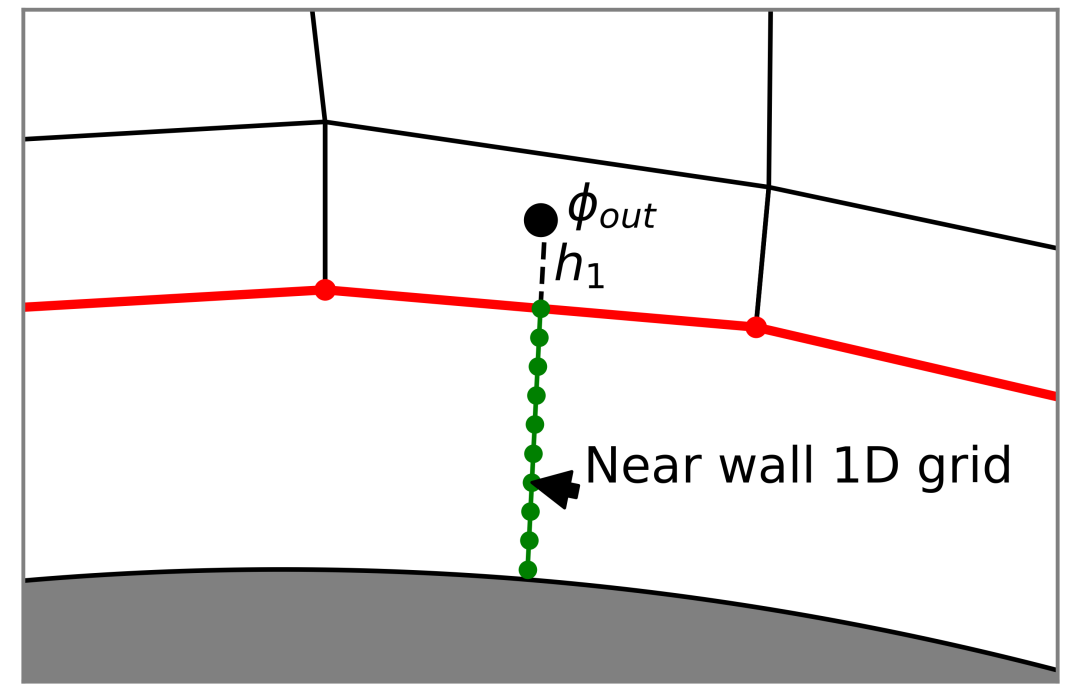

Figure 1: Sketch of the ANDD

The discrete UIBC described above are alternative to the analytical UIBC which are presented in section 2 and based on the local Fourier expansion. Both approaches provide close results provided that a sufficient number of eigenfunctions are taken into account. Numerical experiments show that for quasi-steady flows it is sufficient to take one eigenfunction, while for essentially unsteady flows no more than three eigenfunctions are needed.

The described UIBC are applied for most unknown variables: tangential velocity $u$, temperature $T$ and turbulent variable $\tilde{\nu}$. However, boundary conditions for the normal velocity $v$ is determined in another way. In the following subsections we briefly describe the IBCs for each unknown variable. 


\subsection{Simplified equation for $u$}

The following simplified momentum equation is used for tangential component of velocity:

$$
(\rho u)_{t}=\left(\mu u_{y}\right)_{y}-\frac{d p}{d x}(t)
$$

Term $d p / d x$ is assumed to be constant along the normal to the wall and calculated in the cell adjacent to the interface boundary.

\subsection{Simplified equation for $T, \rho$ profile}

A Robin type boundary condition for the temperature is derived from the simplified energy equation:

$$
T_{t}=\frac{\gamma-1}{\rho R}\left(\lambda T_{y}\right)_{y}+\frac{\gamma-1}{\rho R}\left(u \mu u_{y}\right)_{y}
$$

Here, $u(y)$ profile is substituted from the previous iteration; $R$, gas constant; $\gamma$, specific heat ratio.

Using boundary layer assumption $d p / d y=0$, the density is recalculated via temperature profile from the equation of state:

$$
\rho(y, t)=\frac{p(t)}{R T(y, t)}
$$

Here, $p$ is taken from the cell adjacent to the boundary. It is to be noted that in contrast to the wall functions, the proposed approach allows us to take 
compressibility into account and obtain temperature and density profiles in the near-wall region.

\subsection{Computation of $\tilde{\nu}$ and turbulent viscosity}

There are many approximate expressions for the turbulent viscosity which can be used for direct computations of $\mu_{t}(y)$ and $\tilde{\nu}(y)$ in the near wall domain. We use the approximation from [30]:

$$
\begin{array}{r}
\mu_{t}(\xi)=\mu \kappa \xi^{*}\left[\alpha+\xi^{*}(1-\alpha)^{3 / 2}\right]^{\beta}\left(1-\exp \left(\frac{-\xi^{*}}{1+A \alpha^{*}}\right)\right)^{2}, \\
\kappa=0.41, A=17, \beta=0.78, \alpha=u_{\tau}^{2} / u_{\tau p}^{2}, \xi^{*}=y u_{\tau p} / \mu \\
u_{\tau p}=\sqrt{u_{\tau}^{2}+u_{p}^{2}}, u_{\tau}=\sqrt{\left|\tau_{w}\right| / \rho}, u_{p}=\left|\frac{\mu}{\rho} \frac{d p}{d x}\right|^{1 / 3}
\end{array}
$$

This approximation takes into account the pressure gradient and gives satisfactory accuracy for flows with boundary layer separation.

However, for strongly unsteady flows, approximation (25) is not accurate enough. In such cases IBC for turbulent variable $\tilde{\nu}$ can be obtained from a simplified equation of SA model:

$$
(\rho \tilde{\nu})_{t}=\left(\frac{\rho}{\sigma}(\nu+\tilde{\nu}) \tilde{\nu}_{y}\right)_{y}+S_{s a}(\tilde{\nu}, \nabla \tilde{\nu})
$$

Nonlinear term $S_{s a}$ is linearized with respect to profiles from the previous iteration that leads to form (14). 


\subsection{Boundary condition for normal velocity $v$}

In order to derive a boundary condition for normal component of velocity, let us consider the Taylor expansion for $v$ :

$$
v=a \frac{y^{2}}{2}+b \frac{y^{3}}{6}
$$

From the momentum equation $a=p_{y} /\left.\mu\right|_{y=0}$. Then

$$
v_{y}=a y+b \frac{y^{2}}{2}
$$

Excluding $b$ from these two equations, we obtain a Robin-type boundary condition at $y^{*}$ :

$$
v=\frac{y^{*}}{3} v_{y}+\left.\frac{y^{* 2}}{6} \frac{p_{y}}{\mu}\right|_{y=0}
$$

Here, $p_{y}$ can be taken at $y^{*}$ or set to zero in correspondence to the boundary layer approximation.

\section{Test cases}

\subsection{Pulsating channel flow}

In order to verify accuracy of the developed boundary conditions, we consider a pulsating turbulent channel flow. Despite its relative simplicity, this flow is a good test since it contains essentially unsteady effects such as the Stokes boundary layer, reverse flow, phase shift between wall shear stress 
and inlet velocity $[31,32]$. Another important issue is that steady approaches for wall modeling such as wall functions fail to capture these phenomena [33].

The URANS equations completed by the SA model are solved in 3D rectangular domain with length $L=26 \mathrm{~m}$ and height $H=0.5 \mathrm{~m}$. Since the problem is actually $2 \mathrm{D}$, the three-dimensional hexahedral mesh contains only three cells in the silent $z$ direction. A symmetry boundary condition is imposed on the top boundary and side boundaries. In turn, the boundary conditions of no-slip velocity and constant temperature are specified on the bottom wall boundary. At the inlet, we set the pulsating Reichardt profile $[34]:$

$$
u(y)=u_{r}(y)(1+a \sin (\omega t))
$$

where

$$
u_{r}(y)=u^{*} \frac{1}{\kappa} \ln \left(1+\kappa y^{+}\right)+7.8\left[1-\exp \left(\frac{-y^{+}}{11}\right)-\frac{y^{+}}{11} \exp \left(\frac{-y^{+}}{3}\right)\right],
$$

$y^{+}$is the dimensionless wall distance, $u^{*}$ is the friction velocity, which is set at the inlet.

An IBC for laminar channel flow was considered in [19]. The analytical solution shows that the effect of the unsteadiness in the IBC depends on ratio $y^{* 2} / l_{s}^{2}$, where Stokes length $l_{s}$ is defined by $l_{s}=\sqrt{2 \nu / \omega}$. For quasisteady regime $y^{* 2} / l_{s}^{2} \ll 1$ and intermediate regime $y^{* 2} / l_{s}^{2} \approx 1 \mathrm{SIBC}$ are still applicable. But for high frequencies $y^{* 2} / l_{s}^{2} \gg 1$ only UIBC allows to reach a 
sufficiently accurate solution. These criteria are tested in the current paper in application to a pulsating turbulent channel flow.

The same pattern is observed for RANS equations though the viscosity is not constant. In Figure 2, friction dependence on time is depicted for two regimes: intermediate $\frac{y^{* 2}}{l_{s}^{2}}=1$ and strongly unsteady $\frac{y^{* 2}}{l_{s}^{2}}=6.25$ (in the quasi-steady case both IBC give the same solution). In both computations the intermediate boundary is at $y^{*}=5.4 \times 10^{-3}$, which corresponds to $y^{*+} \approx 100$ for the maximal friction. The vertical lines show $1 / 4$ th and $3 / 4$ ths of pulsation period: in the quasi-steady case both maxima and minima of friction are situated at these positions. For presented regimes a phase shift can be seen.

It is to be noted that unlike the standard wall function approach, even SIBC captures reverse flow and negative friction. This is provided by $d p / d x$ term in the steady model equation, which partially transfers unsteady information from the outer domain.

We found that the use of turbulent viscosity approximations such as [30] for a Dirichlet boundary condition for $\tilde{\nu}$ at the interface boundary $y^{*}$ leads to a very large error in the strongly unsteady case although it works well for steady-state computations. This is why a Robin type IBC is set for $\tilde{\nu}$ parameter.

Figure 3 shows the velocity profiles at $x=20$ at different time moments during one period for a strongly unsteady regime. It can be seen that UIBC allows the reverse flow to be reproduced with high accuracy. Nevertheless, a 

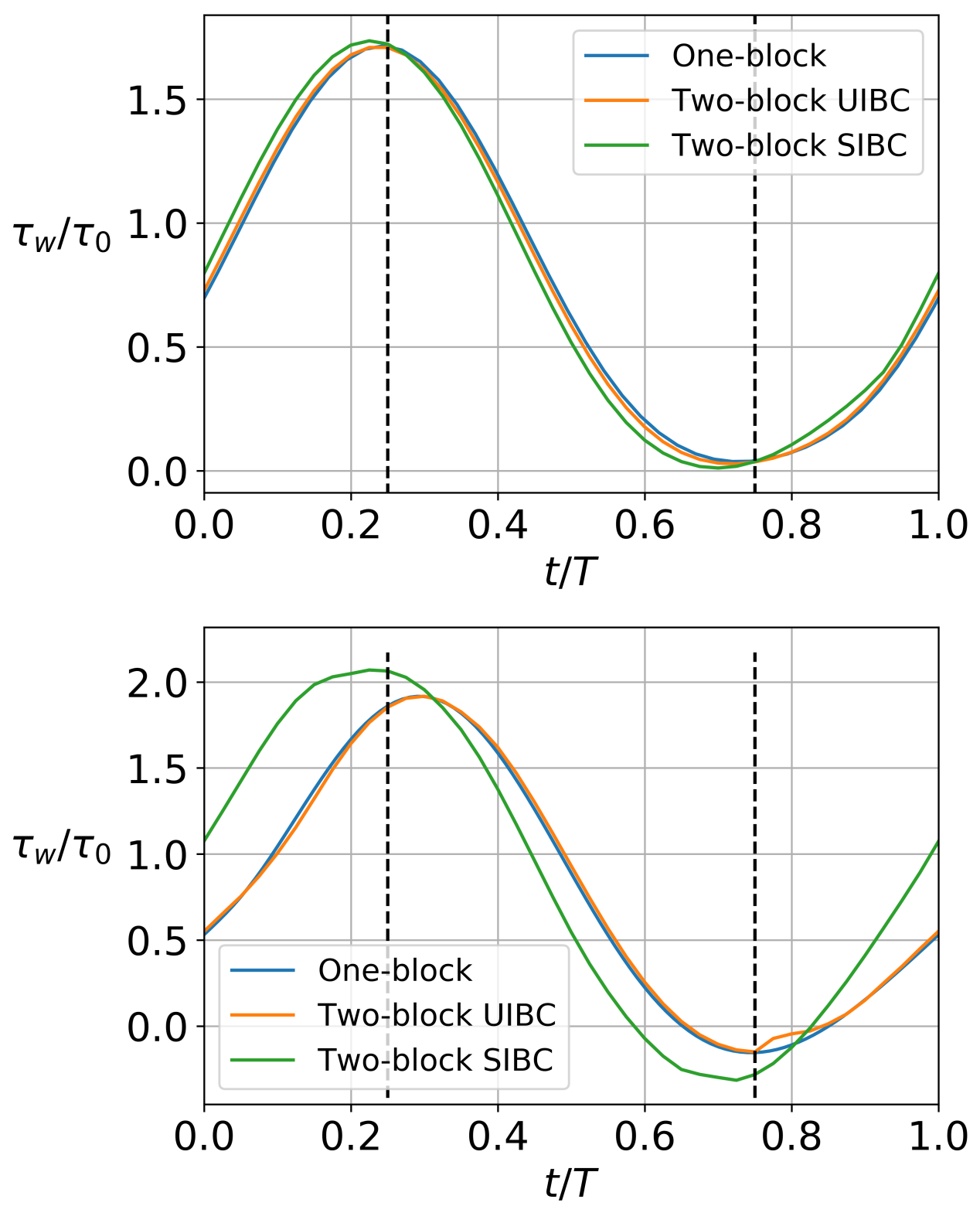

Figure 2: Pulsating channel flow. Dependence of scaled friction on time in one-block and two-block computations for different regimes: top - intermediate regime, bottom essentially unsteady regime 
small discrepancy in the velocity profiles still remains at the interface boundary.
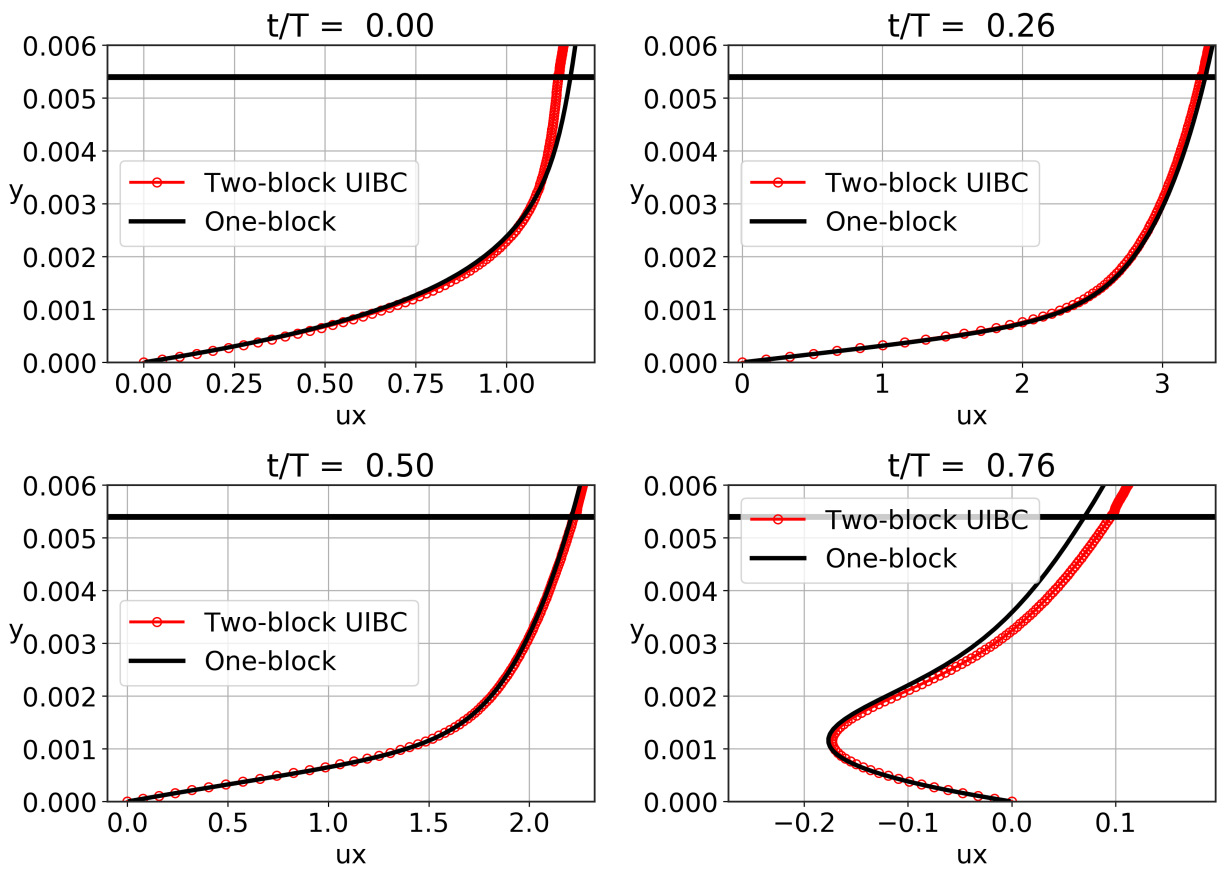

Figure 3: Velocity profiles in pulsating channel flow at different times

\subsection{Pulsating flow past half-cylinder}

The next test case is related to a $2 \mathrm{D}$ pulsating flow past a half-cylinder. The computational domain along with mesh are presented in Figure 4. Similar to the previous test, a 3D mesh was used with three cells along $z$ axis. The symmetry boundary condition is set on the bottom boundary, while the no-slip boundary condition for the velocity and constant temperature are set at the wall. Finally, on the left half of the outer boundary a pulsating flow 
is specified:

$$
u(t)=u_{0}(1+a \sin (\omega t))
$$

with $a=0.8, f=\omega / 2 \pi=30, u_{0}=50 \mathrm{~m} / \mathrm{s}$. On the rest part of outer boundary, we use an outflow boundary condition. In this test case, the freestream Mach number is 0.15 , and Re computed using the cylinder radius equals $6.1 \times 10^{6}$.

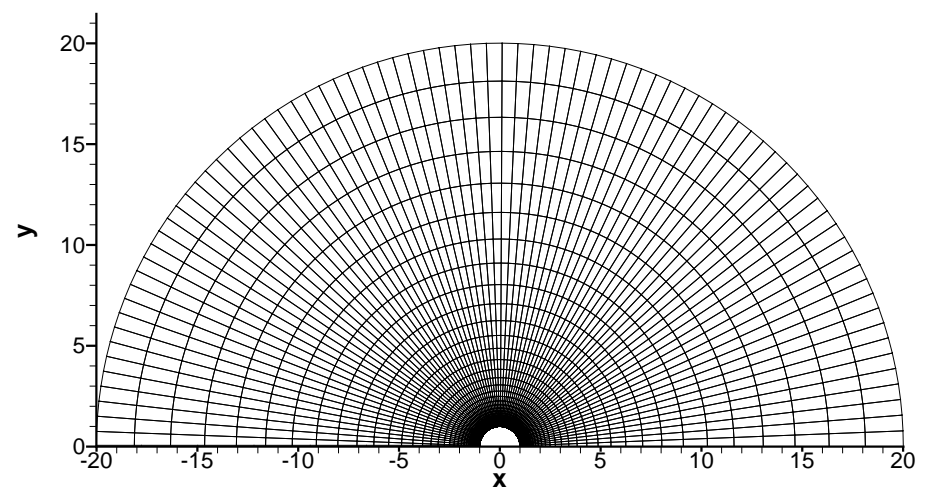

Figure 4: Domain and mesh for half-cylinder test case

Calculations with IBC were carried out for $y^{*}=5 \times 10^{-4}$ that corresponds to the maximum value of $y^{*+} \approx 300$.

Contours of $x$ velocity components for one-block and two-block (outer domain) computations at one time moment are shown at Figure 5. A minor discrepancy occurs in the outer domain. It appears that the approach is quite promising because even if a local 1D solution in the inner domain turns out to be not sufficiently accurate, then an exact decomposition can be applied 
[35] taking the initial approximation from the approximate decomposition.

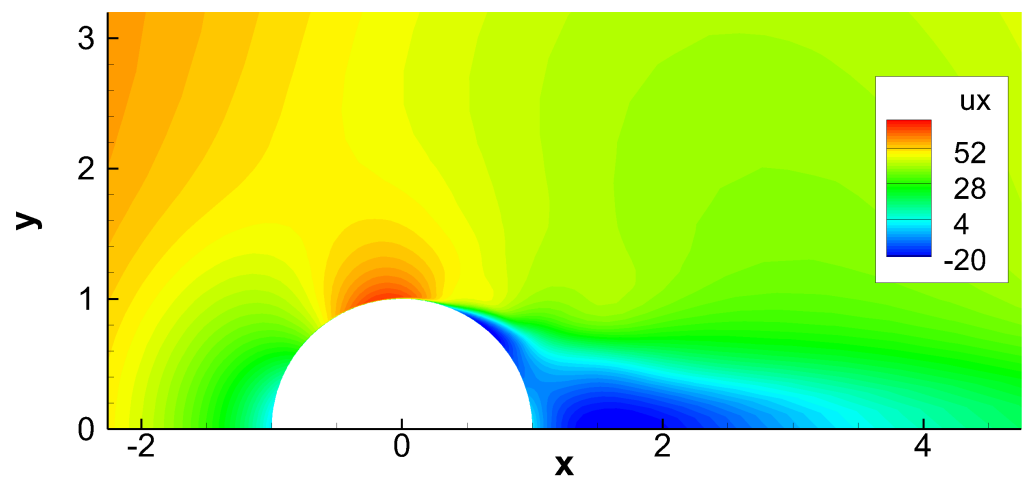

(a) One-block solution

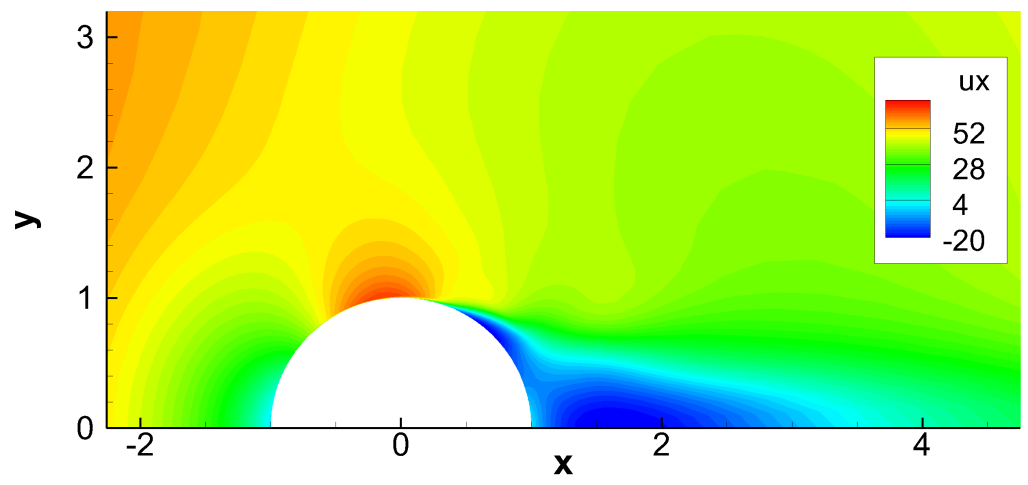

(b) Two-block solution with unsteady IBC

Figure 5: Instantaneous contours of $x$ component of velocity in pulsating flow around half cylinder

Figure 6 shows dependence of the viscous skin friction at the point with $x=-0.17$, where it reaches its maximum. It can be seen, that SIBC leads to a slight phase shift compared with one-block solution. The UIBC demonstrates a good agreement with the reference solution.

Figure 7 shows dependence of the wall friction on the angle measured 


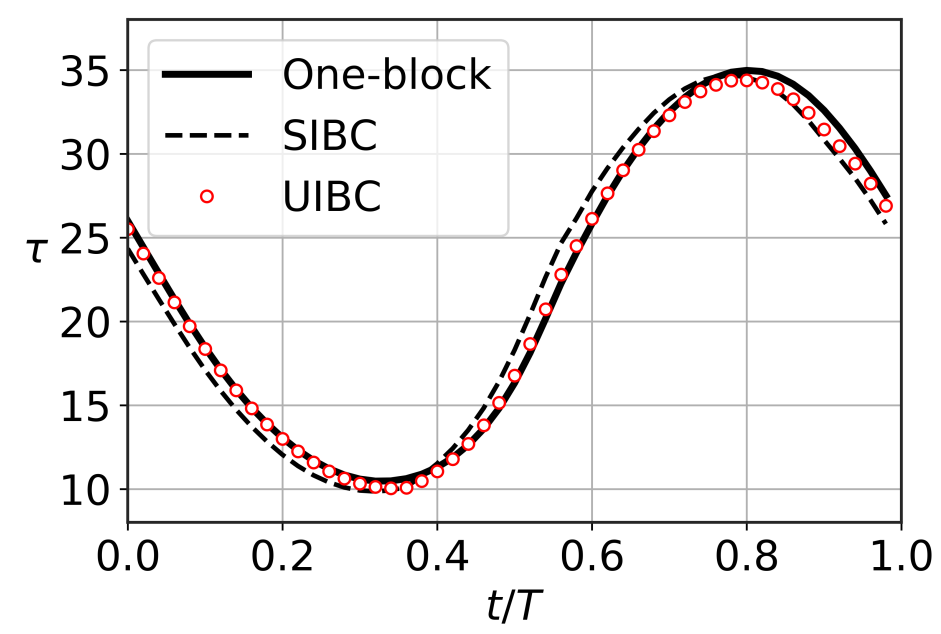

Figure 6: Dependence of surface viscous friction for $x=-0.17$
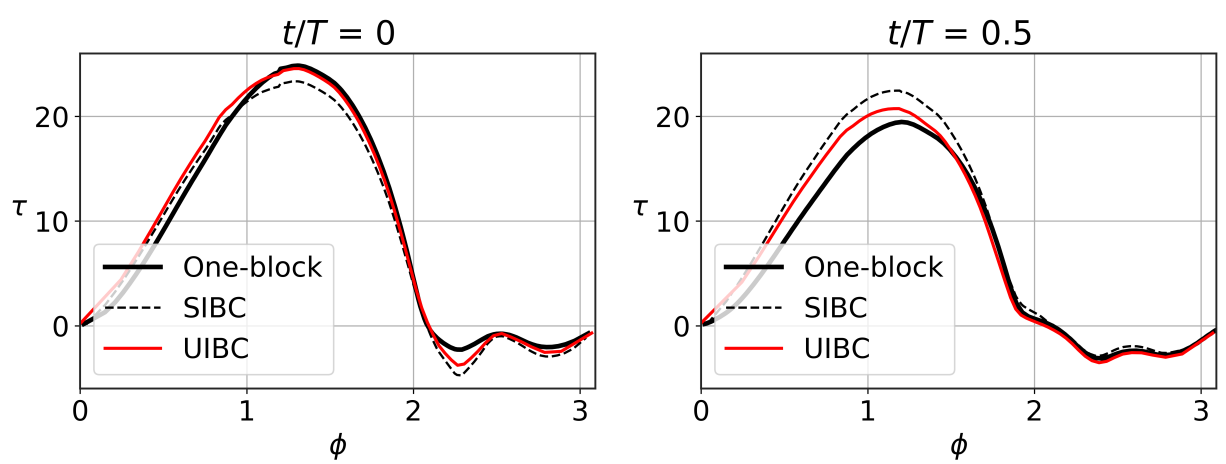

Figure 7: Distribution of the wall friction along cylinder surface for two different times.

from the stagnation point at two different times. Both steady and unsteady IBCs manage to capture the recirculation zone, which is characterized by the negative friction values. A large discrepancy on the front part of the surface is explained by the fact that simplified equations of turbulent boundary layer are no longer valid near the stagnation point. In this area the derivatives, which contain the tangential coordinate and normal velocity, are not small 
anymore.

In the problem in question, $y^{*} / l_{s} \approx 1.6$, therefore, in correspondence with the results from section 6.1, in this regime SIBC are still quite accurate though produce a noticeable phase shift with respect to one-block solution as can be seen in Figures 6 and 7 .

\subsection{Supercritical flow around a circular cylinder at $\mathrm{Re}=10^{7}$}

Next, we consider simulation of subsonic flow around a cylinder with $M=0.15$ and $\operatorname{Re}=10^{7}$. Here, the Reynolds number is calculated via the inlet velocity and cylinder diameter.

A periodic vortex street arises behind the cylinder. The complexity of this test problem is that an unsteady flow separation occurs, which should be captured in order to predict the main quantities: mean drag coefficient and shedding frequency.

It is well known that numerical simulations with different URANS models and LES approach often produce very different results [36]. In this work we focus only on comparison between one-block and two-block solutions.

The problem is solved in a circular domain with radius $25 D$ where $D$ is the diameter of the cylinder.

For one-block computation an unstructured 3D mesh with 250 cells along radial direction, 320 along azimuthal direction and three cells along $z$ axis is used. The normal size of the first near wall cell equals $2.5 \times 10^{-4} D$ which corresponds to maximum $y^{+} \approx 1$. For time marching approximately 300 
steps for one period is used.

In Figure 8 comparison of streamlines and velocity magnitude for both solutions is shown. The two-block computation with $y^{*+} \approx 100$ gives a solution close to the benchmark one-block solution with a slightly different form of separation zones. It is interesting that for this test both IBC and UIBC produce very close results. This test can be classified as the intermediate regime in terms of $y^{*+} / l_{s}$ ratio.
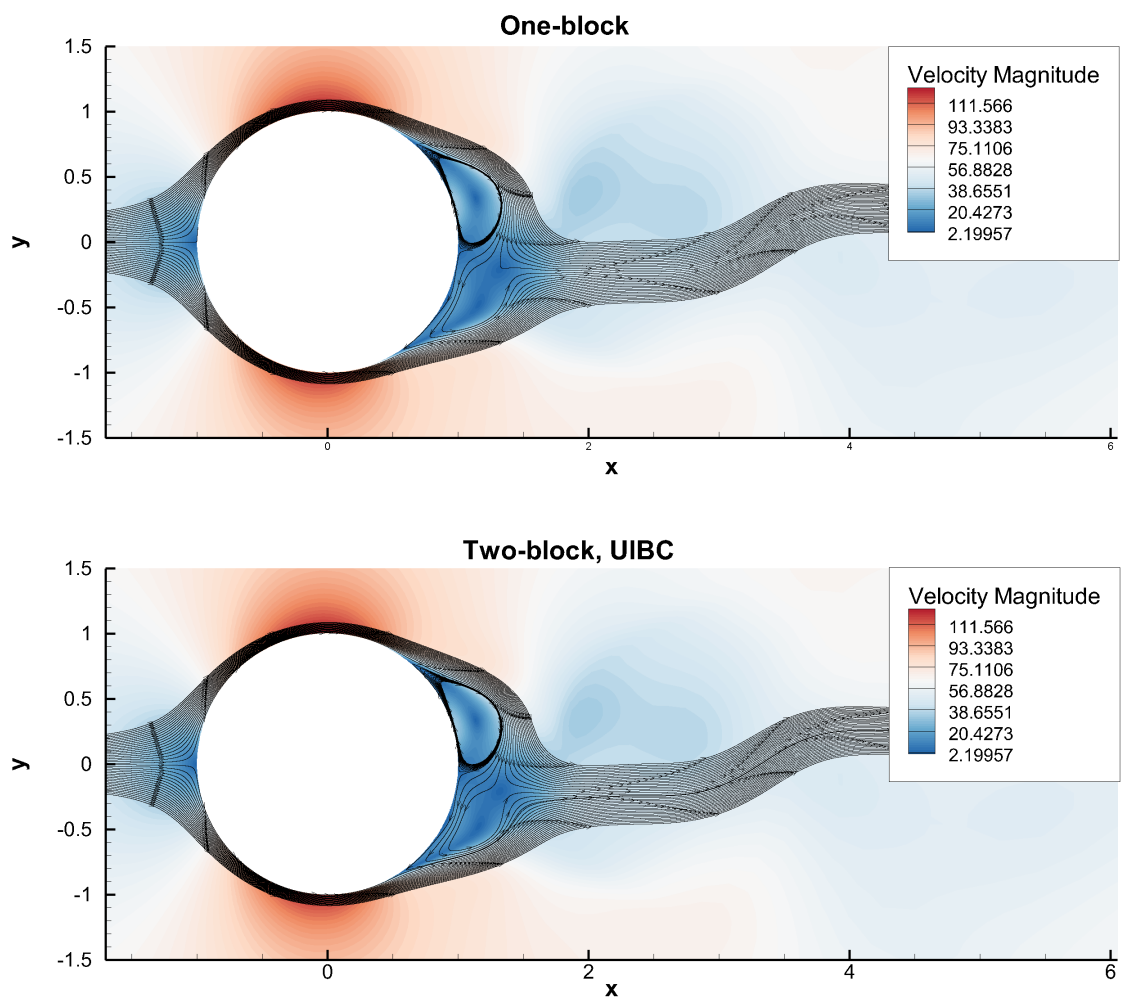

Figure 8: Comparison of one-block and two-block solutions at the moment of maximal lift

Mean drag coefficient $\bar{C}_{D}$, Strouhal number $S t=f D / u_{\infty}(f$ - shedding frequency) and root mean square of the lift coefficient $C_{L}^{r m s}$ are presented 
in table 1. Two-block solution accurately reproduces shedding frequency, however there is a discrepancy in mean drag like in the previous test case.

\begin{tabular}{|c|c|c|c|}
\hline & $S t$ & $\bar{C}_{D}$ & $C_{L}^{\text {rms }}$ \\
\hline One-block & 0.278 & 0.42 & 0.31 \\
\hline Two-block & 0.280 & 0.37 & 0.10 \\
\hline
\end{tabular}

Table 1: Main quantities for the vortex shedding test

\section{Conclusion}

In this paper unsteady interface boundary conditions for URANS equations have been presented. The boundary conditions are derived by transferring the wall boundary condition to an interface boundary using a domain decomposition approach. The UIBC contains a memory term to be taken into account for essentially unsteady problems. The UIBCs have been implemented in a 3D unstructured-mesh solver for compressible URANS equations and applied for numerical solution of unsteady problems. Numerical experiments show that the UIBCs allow unsteady phenomena to be captured and yield satisfactory prediction of the skin friction for the location of the interface boundary up to $y^{*+}=300$. It has been shown that the unsteady memory terms in the IBCs should be taken into account in essentially unsteady problems. The criteria for essential unsteadiness in the IBCs, earlier obtained for the laminar case, have been confirmed for turbulent flows. The obtained results can be used for URANS-LES hybrid models in the future. 


\section{Acknowledgement}

The work was supported by the Russian Science Foundation (Project N 18-19-00098).

Some of the calculations were run on supercomputers of Joint Supercomputing Center of the Russian Academy of Sciences.

\section{In memoriam}

This paper is dedicated to the memory of Dr. Douglas Nelson Woods (*January $11^{\text {th }} 1985$ - †September $\left.11^{\text {th }} 2019\right)$, promising young scientist and postdoctoral research fellow at Los Alamos National Laboratory. Our thoughts and wishes go to his wife Jessica, to his parents Susan and Tom, to his sister Rebecca and to his brother Chris, whom he left behind.

\section{References}

[1] T. Knopp, On grid-independence of RANS predictions for aerodynamic flows using model-consistent universal wall-functions, in: Proceedings of ECCOMAS CFD, 2006.

[2] S. Patankar, D. Spalding, Heat and mass transfer in boundary layers, Morgan-Grampian, 1968.

[3] P. Durbin, Limiters and wall treatments in applied turbulence modeling, Fluid Dynamics Research 41 (2009). 
[4] H. Grotjans, F. Menter, Wall functions for industrial applications, in: K. Papailiou (Ed.), Proceedings of Computational Fluid Dynamics'98, ECCOMAS, Chichester, UK: John Wiley \& Sons, 1998, pp. 1112-7.

[5] G. Kalitzin, G. Medic, G. Iaccarino, P. Durbin, Near-wall behavior of RANS turbulence models and implications for wall functions, Journal of Computational Physics 204 (2005) 265-291.

[6] T. Craft, S. Gant, H. Iacovides, B. Launder, A new wall function strategy for complex turbulent flows, Numerical Heat Transfer, Part B: Fundamentals 45 (2004) 301-318.

[7] S. Utyuzhnikov, Some new approaches to building and implementation of wall-functions for modeling of near-wall turbulent flows, Computers and Fluids 34 (2005) 771-784.

[8] S. Utyuzhnikov, Generalized wall functions and their application for simulation of turbulent flows, International Journal for Numerical Methods in Fluids 47 (2005) 1323-1328.

[9] S. Utyuzhnikov, The method of boundary condition transfer in application to modeling near-wall turbulent flows, Computers and Fluids 35 (2006) 1193-1204.

[10] S. Utyuzhnikov, Robin-type wall functions and their numerical implementation, Applied Numerical Mathematics 58 (2008) 1521-1533. 
[11] S. Utyuzhnikov, Generalized Calderón-Ryaben'kii's potentials, IMA Journal of Applied Mathematics (Institute of Mathematics and Its Applications) 74 (2009) 128-148.

[12] S. Utyuzhnikov, Interface boundary conditions in near-wall turbulence modeling, Computers and Fluids 68 (2012) 186-191.

[13] S. Utyuzhnikov, Domain decomposition for near-wall turbulent flows, Computers and Fluids 38 (2009) 1710-1717.

[14] S. Utyuzhnikov, Towards development of unsteady near-wall interface boundary conditions for turbulence modeling, Computer Physics Communications 185 (2014) 2879-2884. doi:10.1016/j.cpc.2014.07.009.

[15] A. Jones, S. Utyuzhnikov, Application of a near-wall domain decomposition method to turbulent flows with heat transfer, Computers and Fluids 119 (2015) 87-100.

[16] A. Jones, S. Utyuzhnikov, A near-wall domain decomposition approach in application to turbulent flow in a diffuser, Applied Mathematical Modelling 40 (2016) 329-342.

[17] A. Jones, S. Utyuzhnikov, Efficient computation of turbulent flow in ribbed passages using a non-overlapping near-wall domain decomposition method, Computer Physics Communications 217 (2017) 1-10.

[18] S. Utyuzhnikov, M. Petrov, A. Chikitkin, V. Titarev, On extension of near-wall non-overlapping domain decomposition to essentially unsteady 
turbulent flows, Smart Innovation, Systems and Technologies 133 (2019) 199-209.

[19] S. Utyuzhnikov, N. Smirnova, Unsteady interface boundary conditions for near-wall turbulence modeling, Computers and Mathematics with Applications 79 (2020) 1483-1502.

[20] V. S. Vladimirov, Equations of Mathematical Physics, Dekker, New York, 1971.

[21] L. Eça, M. Hoekstra, A. Hay, D. Pelletier, A manufactured solution for a two-dimensional steady wall-bounded incompressible turbulent flow, International Journal of Computational Fluid Dynamics 21 (2007) 175188.

[22] M. Petrov, A. Tambova, V. Titarev, S. Utyuzhnikov, A. Chikitkin, Flowmodellium software package for calculating high-speed flows of compressible fluid, Computational Mathematics and Mathematical Physics 58 (2018) 1865-1886.

[23] V. Kolgan, Application of the principle of minimizing the derivative to the construction of finite-difference schemes for computing discontinuous solutions of gas dynamics, J. Comput. Phys. 230 (2011) 2384-2390.

[24] E. Toro, M. Spruce, W. Speares, Restoration of the contact surface in the Harten-Lax-van Leer Riemann solver, Journal of Shock Waves 4 (1994) 25-34. 
[25] E. Toro, Riemann solvers and numerical methods for fluid dynamics, third ed., Springer-Verlag, 2009.

[26] M. Petrov, V. Titarev, S. Utyuzhnikov, A. Chikitkin, A multithreaded openmp implementation of the LU-SGS method using the multilevel decomposition of the unstructured computational mesh, Computational Mathematics and Mathematical Physics 57 (2017) 1856-1865.

[27] A. Chikitkin, M. Petrov, V. Titarev, S. Utyuzhnikov, Parallel versions of implicit LU-SGS method, Lobachevskii Journal of Mathematics 39 (2018) 503-512.

[28] S. Yoon, A. Jameson, Lower-upper symmetric-gauss-seidel method for the euler and navier stokes equations, AIAA Journal 26 (1988) 10251026.

[29] I. Men'shov, Y. Nakamura, An implicit advection upwind splitting scheme for hypersonic air flows in thermochemical nonequilibrium, in: A Collection of Technical Papers of 6th Int. Symp. on CFD, volume 2, Lake Tahoe, Nevada, 1995, p. 815.

[30] C. Duprat, G. Balarac, O. Métais, P. M. Congedo, O. Brugière, A wall-layer model for large-eddy simulations of turbulent flows with/out pressure gradient, Physics of Fluids 23 (2011) 015101.

[31] P. R. Spalart, B. S. Baldwin, Direct simulation of a turbulent oscillating boundary layer, in: J.-C. André, J. Cousteix, F. Durst, B. E. 
Launder, F. W. Schmidt, J. H. Whitelaw (Eds.), Turbulent Shear Flows 6, Springer Berlin Heidelberg, Berlin, Heidelberg, 1989, pp. 417-440.

[32] S. Tardu, G. Binder, R. Blackwelder, Turbulent channel flow with largeamplitude velocity oscillations, Journal of Fluid Mechanics 267 (1994) 109-151. doi:10.1017/S0022112094001138.

[33] D. Panara, M. Porta, T. Schoenfeld, LES and URANS unsteady boundary layer strategies for pulsating and oscillating turbulent channel flow applications, in: Proceedings ECCOMAS CFD, 2006.

[34] H. Reichardt, Vollständige darstellung der turbulenten geschwindigkeitsverteilung in glatten leitungen, ZAMM-Journal of Applied Mathematics and Mechanics/Zeitschrift für Angewandte Mathematik und Mechanik 31 (1951) 208-219.

[35] S. Utyuzhnikov, C. Wang, Exact non-overlapping domain decomposition for near-wall turbulence modeling, Computers and Fluids 181 (2019) 283-291. doi:10.1016/j.compfluid.2019.02.005.

[36] C. Moussaed, M. Vittoria Salvetti, S. Wornom, B. Koobus, A. Dervieux, Simulation of the flow past a circular cylinder in the supercritical regime by blending RANS and variational-multiscale LES models, Journal of Fluids and Structures 47 (2014) 114-123. 